\title{
Pathological evaluation of computed tomography images of lungs
}

\author{
ROSEMARY CODDINGTON, $\uparrow$ SL MERA, PR GODDARD, * JWB BRADFIELD \\ From the University Departments of Pathology and *Radiodiagnosis, Bristol Roval Infirmary, Bristol \\ BS2 $8 H W$
}

SUMmARY A method is described which allows the features seen in images generated during computed tomography (CT) of lungs previously removed at necropsy to be compared with those seen in corresponding thin sections made from the same lungs. After removal from the body, lungs were inflation-fixed using warm formalin vapour before being $x$-rayed and then scanned in the inflated state. Slices corresponding to the CT scan images were made and $x$-rayed. Paper mounted sections were then prepared from each slice. Using these methods pathological correlative studies can be used both to validate the interpretation of CT scans of lungs and to assess the sensitivity of this imaging technique.

Computed tomography (CT) is increasingly used in the investigation of lung disease. ${ }^{-3}$ Confidence in the interpretation of the images generated can only be acquired by adequate correlative studies. In this hospital three approaches are currently under intensive exploration. Firstly, we are comparing the results of CT scanning with the results of lung function tests. ${ }^{4}$ Secondly, we are documenting the appearances of standardised phantoms used to simulate lung structures. ${ }^{6}$ Thirdly, we are comparing scan images with the detailed structure of both normal and known pathological lesions in lungs removed at necropsy. This paper presents the experience gained by the third approach, which provides information about the validity of interpretation of lung pathology as seen in the images generated by computed tomography of lungs.

\section{Material and methods}

REMOVAL AND FIXATION OF LUNGS

Intact lungs were removed at necropsy with special care to avoid damage to the pleura. After removal, each pair of lungs was fixed by inflation with formalin vapour. ${ }^{7}$ In brief, the lungs were suspended in a perspex box by a tracheal cannula above aqueous formaldehyde solution heated to $40^{\circ} \mathrm{C}$. An

† Present address: Department of Histopathology, Southampton General Hospital, Hants.

Accepted for publication 24 September 1981 external pump provided intermittent inflation with formalin vapour for approximately $24 \mathrm{~h}$.

At the beginning and end of the procedure, anterior-posterior and lateral radiographs were taken of the lungs within the box. By comparing the appearances in these two sets of radiographs it was possible to detect any unexpanded areas or regions of fluid accumulation. Correlation with any antemortem radiographs was also possible. Before removal of the lungs from the fixation apparatus a metal clamp was tightened across the trachea to prevent deflation.

\section{COMPUTED TOMOGRAPHY}

This was performed within two hours of cessation of inflation-fixation. The inflated lungs were placed in the supine position within a plastic bucket, enclosed in a polythene bag, and the intervening space packed with EMI "bean bags" (Fig. 1). Scans were obtained in $13 \mathrm{~mm}$ contiguous slices, commencing at the base, using an EMI CT 5005 Series 2 scanner with the SW 2085 software system and extended dynamic range. Two phantoms comprising perspex tubes containing air and water respectively were included in each scan in order that variations in attenuation coefficients between scans could be detected.

SLICING OF LUNGS

After scanning, the lungs were sliced transversely $\underset{\gamma}{ }$ at exactly $13 \mathrm{~mm}$ intervals, using a Berkel GF 30 


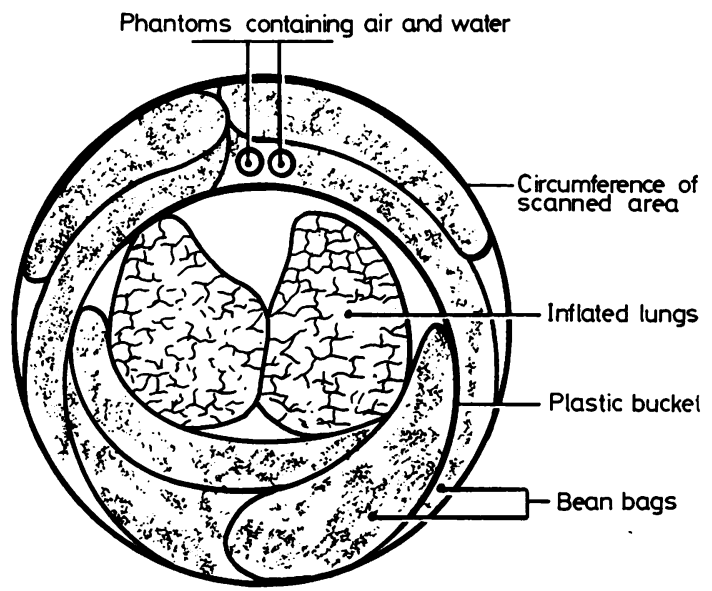

Fig. 1 Diagram to show inflated lungs packed within a plastic bucket to provide the optimal conditions for computed tomography.

slicing machine, starting at the base of the lower lobes. Slices were arranged to correspond as nearly as possible to the slices of the CT scan.

\section{RADIOLOGY OF SLICES}

The lung slices were $x$-rayed using a Senograph mammography unit at $20 \mathrm{kv}, 20 \mathrm{~mA}$ and six seconds with $3 \mathrm{M}$ type-S non-screen film. The technique was that described previously for the study of breast slices. ${ }^{8}$

PREPARATION OF PAPER MOUNTED SECTIONS Paper mounted sections were prepared using a modification of the method of Gough and Wentworth. ${ }^{9}$ In short, each complete $13 \mathrm{~mm}$ lung slice was washed in running tap water for $48 \mathrm{~h}$ to remove the formalin, and was then placed in two successive changes of gelatin solution A and incubated for $24 \mathrm{~h}$ and $48 \mathrm{~h}$ respectively at $45^{\circ} \mathrm{C}$. The specimen was allowed to cool on the block holder of a large sledge microtome and then frozen overnight at $-20^{\circ} \mathrm{C}$. Frozen sections were cut at $300 \mu \mathrm{m}$ thickness on an MSE large section microtome. Cut sections were spread out in a pool of warm gelatin solution $B$ on a perspex sheet and covered with a sheet of Whatman No 1 filter paper. A soft rubber roller was used to remove air bubbles and surplus gelatin before drying the preparation in a Durst FRC 400 photographic print dryer for $24 \mathrm{~h}$. The paper mounted section could then be peeled away from its backing perspex sheet. were immersed in $10 \%$ formol saline for at least $24 \mathrm{~h}$. After processing, embedding in paraffin, and cutting, $4 \mu \mathrm{m}$ sections were stained using standard procedures for examination by light microscopy. This allowed assessment of the quality of preservation of the lung tissue and microscopic confirmation of features seen by naked eye in the mounted sections.

\section{Results}

PREPARATION OF LUNGS

The procedure for inflation-fixation produced good tissue preservation in an inflated state with normal, emphysematous and bronchiectic lungs. Radiographs taken after fixation but before removal of the lungs from the apparatus showed that inflation was uniform throughout all lobes and that there was no fluid accumulation in the bases. Predictably, inflation and consequent fixation were unsatisfactory in the one case of fibrosing alveolitis which was studied, By clamping the trachea at the end of fixation it was possible to maintain the lungs in their inflated state throughout the period of CT scanning and subsequent slicing.

NORMAL LUNGS

\section{Scanning}

The attenuation of the $x$-ray beam during passage through isolated inflated lungs is much lower than during passage through a normal intact human thorax due to both the inherent low density of lung tissue and the lack of any surrounding thoracic wall. Because of this, over-ranging of the detectors on the CT scanner could only be prevented by providing artificial packing material round the lungs. In pilot studies the results using EMI "bean bags" as packing (Fig. 1) proved much better than those with water or gelatin. The whole preparation within the bucket was kept within the area being scanned.

Under these conditions, computed tomography of post-mortem lungs after removal from the body produced images of similar quality (Fig. 3a) to those of normal lungs in life (Fig. 2) and the pulmonary vasculature, airways and lung parenchyma were each displayed in comparable detail by either technique. The mean attenuation values for the lung fields was very similar from post-mortem lungs as from lungs in life. Even so, because some alteration in this value for blood vessels and parenchyma occurred during formalin fixation we chose at this stage not to embark on any attempt to measure regional variations using these isolated fixed lungs.

Lung slices

By setting the slicing machine to cut at $13 \mathrm{~mm}$ 


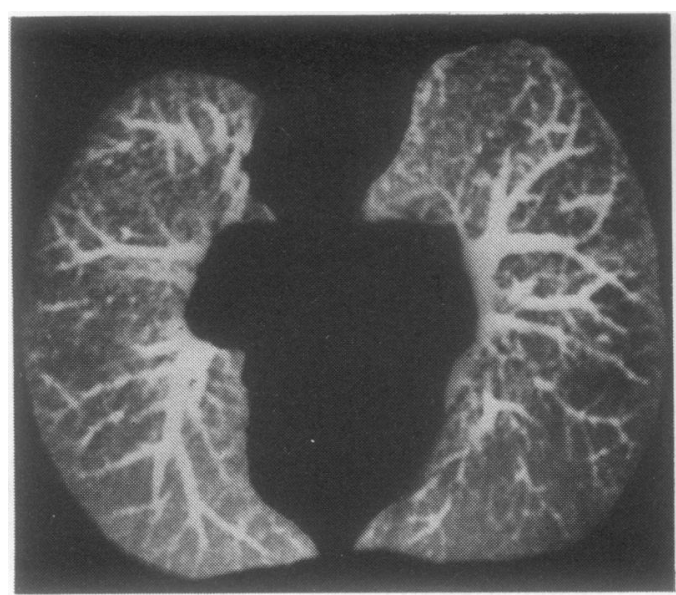

Fig. 2

Fig. 2 CT scan of lungs of a normal person during life.

Fig. 3 (a) CT scan of previously removed normal lungs, inflation-fixed before scanning; $(b) x$-ray of $a$ separated $13 \mathrm{~mm}$ axial slice of lung tissue corresponding to that seen in $(a) ;(c)$ mounted thin section of lungs taken from the slice shown in $(a)$ and $(b)$.

intervals it was possible to prepare lung slices which corresponded very closely to the axial images obtained by computed tomography. The method used here for radiography of the slices produced high resolution pictures (Fig. 3b) thereby providing an ideal method for comparison with the CT scan images in that both techniques visualised the full $13 \mathrm{~mm}$ depth of each slice. In addition the Gough paper mounted sections (Fig. 3c) allowed final validation of interepretation, but at the expense of depth, in that these sections are only $300 \mu \mathrm{m}$ in thickness. Four micron sections confirmed that preservation of the tissue was adequate. By using the above techniques in sequence on the same lungs it was possible to show that where small blood vessels are followed out towards the periphery on CT scans, seven orders of branching are detectable; this corresponds to order number 11 of pulmonary arterial branching in Cummings' nomenclature. ${ }^{10}$ Study of the corresponding Gough sections revealed that the smallest blood vessels which can be resolved on CT scans are of the order of $1 \mathrm{~mm}$ in diameter.

EMPHYSEMATOUS LUNGS (Figs $4 a, 4 b, 4 c$ )

Areas of lung which had been interpreted as emphysematous on CT scan images were confirmed as emphysematous in the Gough sections. However, the latter technique showed that computed tomography was not able to resolve some of the thin septae
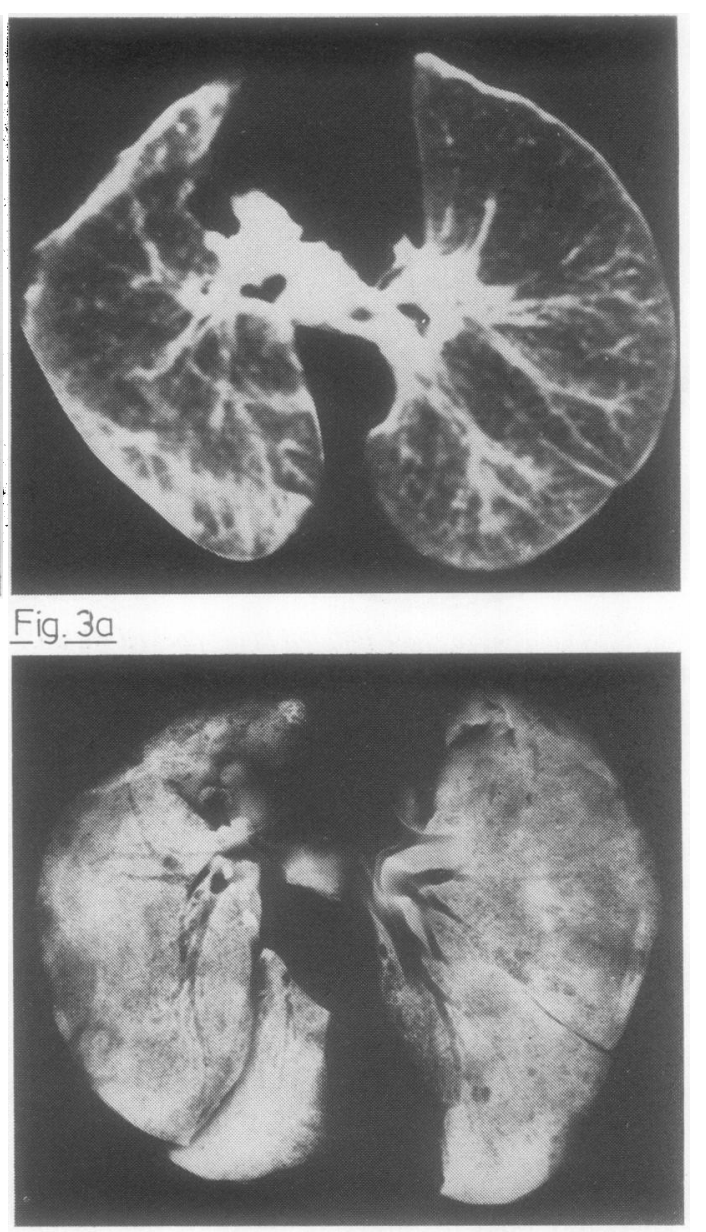

Fig. 3 b

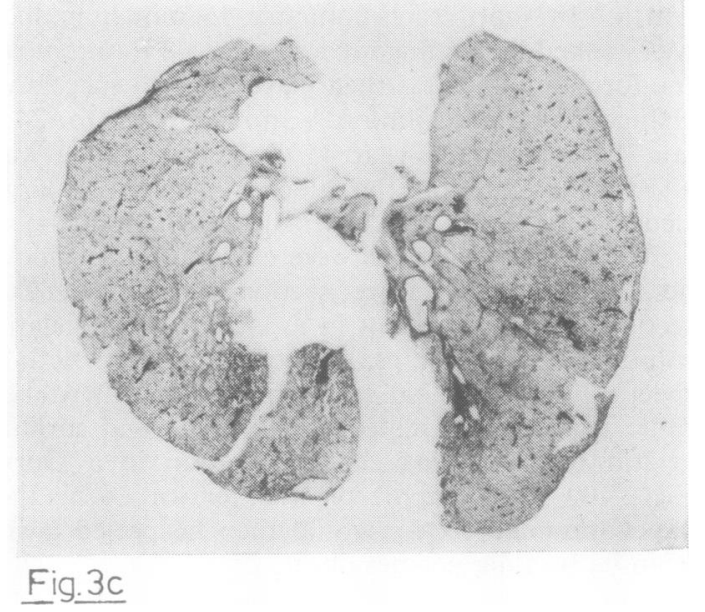




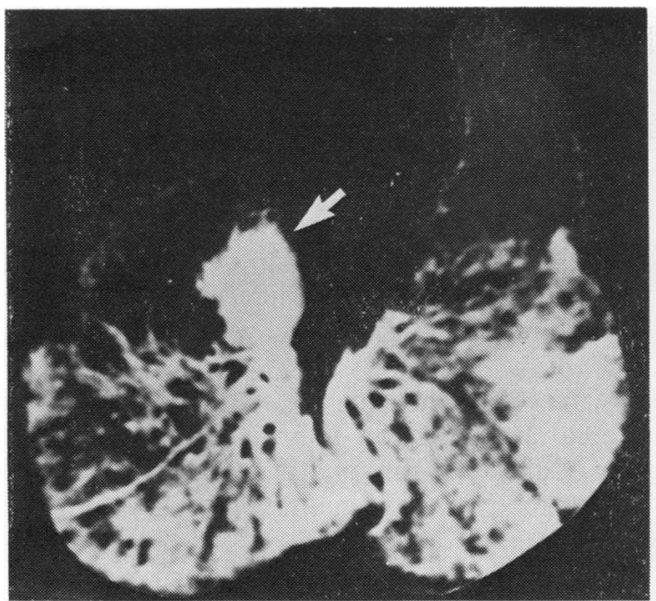

Fig. 4 a

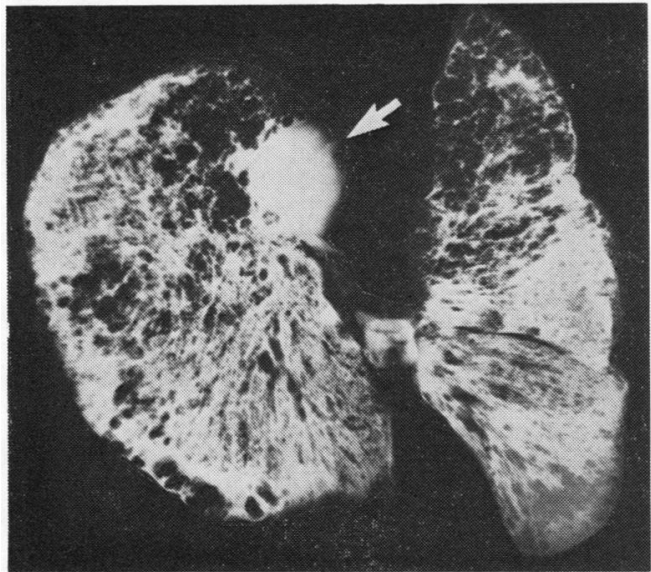

Fig. $4 \mathrm{~b}$

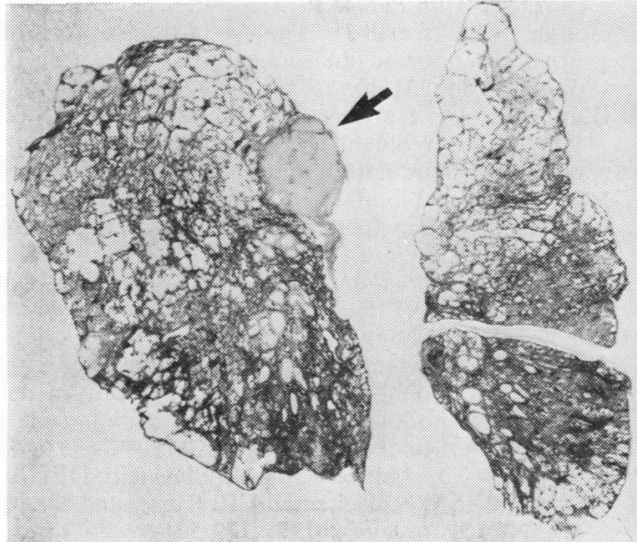

Fig. $4 \mathrm{c}$

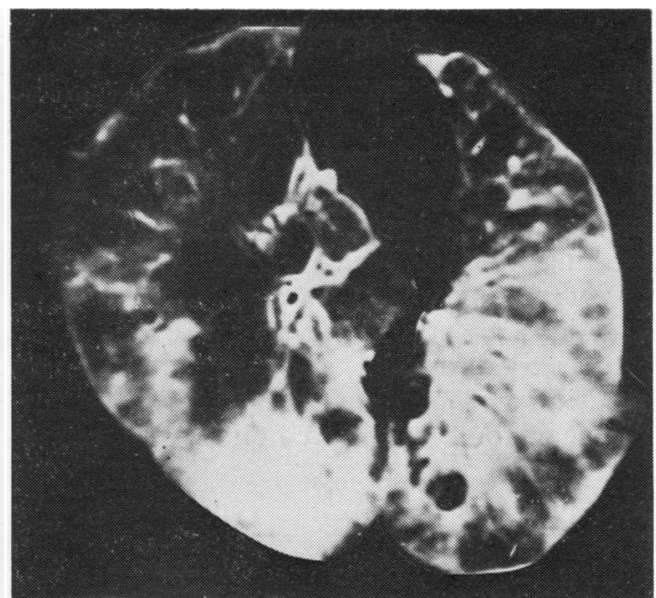

Fig. $5 a$

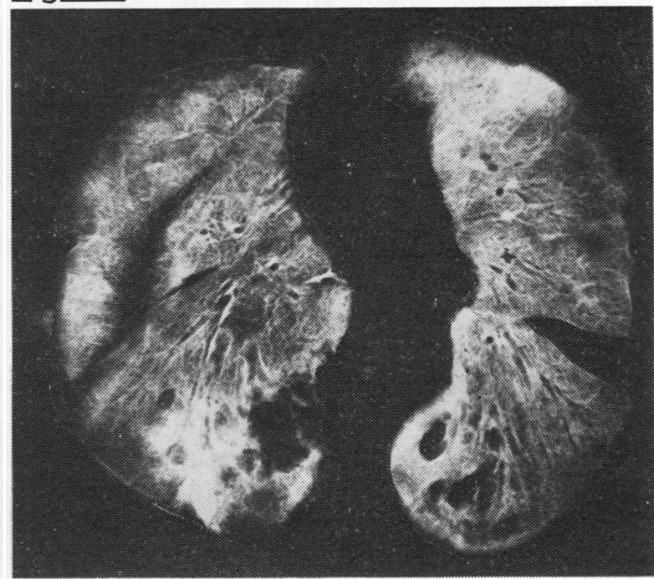

Fig. $5 b$

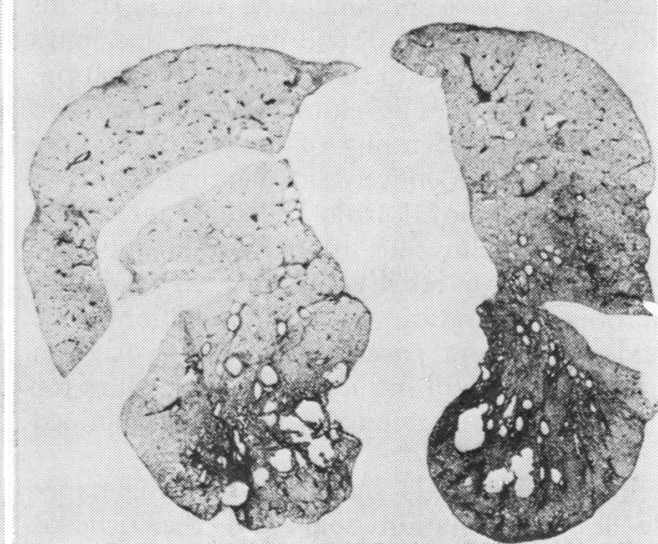

Fig. $5 c$

Fig. 4 (a) CT scan of previously removed emphysematous lungs, inflation-fixed before scanning. A carcinoma is also present (arrow); (b) $x$-ray of separated $13 \mathrm{~mm}$ axial slice of lung tissue corresponding to that seen in (a); (c) mounted thin section of lungs taken from the slice shown in (a) and (b).

Fig. 5 (a) CT scan of previously removed bronchiectatic lungs, inflation-fixed before scanning; $(b) x$-ray of separated $13 \mathrm{~mm}$ axial slice of lung tissue corresponding to that seen in $(a) ;(c)$ mounted thin section of lungs taken from the slice shown in $(a)$ and $(b)$. 
within large areas of severe panacinar emphysema. This sequence of pictures also shows the accurate imaging of a central carcinoma in the right lung.

BRONCHIECTATIC LUNGS (Figs 5a, 5b, 5c)

Areas of lung which had been interpreted as bronchiectatic were always confirmed as such in the Gough sections. By comparing the size of the individual enlarged bronchial sacs using the three techniques it was shown that sacs of greater than $3 \mathrm{~mm}$ diameter could be resolved on the CT scan.

\section{Discussion}

Computed tomography offers great potential in the investigation of the patient with regional pathology in the lung. Several previous studies have compared the value of conventional chest radiography or tomography with that of CT scanning but most of these have lacked pathological validation of their interpretation of individual lesions. However, Schaner et $a l^{11}$ compared CT scans with conventional tomography in a study of nodules in lungs which were then examined pathologically after resection. The results showed that the lower limit for detection of parenchymal nodules was $3 \mathrm{~mm}$ by the former technique, against $6 \mathrm{~mm}$ by the latter. Rosenblum et $a l^{12}$ performed CT scans on four emphysematous patients and related the images to the results of both lung function tests and conventional chest radiography. The advantage of the present method is that it allows direct correlations to be made between corresponding lung radiography, CT scanning, radiography of lung slices and mounted thin sections of the same lung. The success of this depends on the ability to obtain fixed inflated lungs which have not filled with fluid during preparation. For this, the techniques developed for the validation of conventional radiography of lungs, ${ }^{7}$ or of lung slices, ${ }^{13}$ proved to be entirely satisfactory. Scanning the lungs after removal from the body presented some initial problems due to over-ranging of the detectors but this was resolved by effective bolussing; thus, although alterations in the attenuation value of blood vessels and parenchyma due to fixation were noted, the overall mean attenuation values for the lung fields were similar to those obtained in life.

The illustrations of CT scans shown in this paper provide less information than did the original computer-generated images in which a range of structures of different densities could be brought into contrast by alteration of the window and the level to allow adjustment of the variable grey scale appropriate to the particular structures under observation.
An important application of the methods described here is the quantification of sensitivity of CT scan techniques. These preliminary studies already allow an estimation to be made of the level of resolution of vessels and bronchiectactic bronchi. Studies are currently being made to find out whether degrees of regional abnormality in different parts of the lung can be reliably measured.

We are grateful to the Bristol Health District Research Fund for financial support. We thank $\mathrm{Mr}$ Cliff Jeal for photography and Miss Vicky Thyer for typing the manuscript.

\section{References}

${ }^{1}$ Kreel L. Computed tomography of the thorax. Radiol Clin North Am 1978;16:575-84.

${ }^{2}$ Siegelman SS. Computed tomography. In: Siegelman SS. Stitik FP, Sumner WR, eds. Pulmonary system: practical approaches to pulmonary diagnosis. New York Grune and Stratton, 1979:91-121.

${ }^{3}$ Heitzman ER. Computed tomography of the thorax Am J Roentg $1981 ; 136: 2-12$.

${ }^{4}$ Goddard PR, Nicholson EM, Todd JR, Watt I, Laszlo (j. Normal and emphysematous lungs studied by computerised axial tomography. Thorax 1980;35:236.

${ }^{5}$ Nicholson EM. Goddard PR, Todd J, Watt I, Laszlo G. Severity and patterns of distribution of pulmonary emphysema in patients with chronic airflow obstruction shown by computerised tomography. Clin Sci 1980. 59:30P.

${ }^{6}$ Goddard PR. In preparation.

7 Wright BM, Slavin G, Kreel L, Callan K, Sandin B. Post mortem inflation and fixation of human lungs. A technique for pathologic and radiologic correlations. Thorax 1974;29:189-94.

${ }^{8}$ Davies JD, Roberts G, Richardson PJ. A serial whole organ slicing technique for examining surgically resected breasts. J Clin Pathol 1973;26:891-2.

${ }^{9}$ Gough J, Wentworth JE. The use of thin sections of entire organs in morbid anatomical studies. $J$ Roy Microsc Soc 1949;69:231-40.

${ }^{10}$ Cummings G, Harding LK, Horsfield K, Prowse K. Singhal SS, Woldenberg. Morphological aspects of the pulmonary circulation and of the airways. In: Fluid dynamics of blood circulation and respiratory flow Advisory Group for Aerospace Research and Develop ment; NATO Conf Proc No 65. In: Harris P, Heath D The human circulation. 2nd ed. Edinburgh: Churchill Livingstone, 1977:6.

${ }^{11}$ Schaner EG, Chang AE, Doppman JL, Conkie DM Flye MW, Rosenburg SA. Comparison of computed and conventional whole lung tomography in detecting pulmonary nodules: a prospective radiological pathologic study. Am J Roentg 1978;131:51-4.

12 Rosenblum LJ, Mauceri RA, Wellenstein DE, Bassario DA, Cohen WN. Heitzman ER. Computed tomography of the lung. Radiology 1978:129:521-4.

${ }^{13}$ Crow J, Slavin G. Kreel L. Pulmonary metastasis: a pathologic and radiologic study. Cancer 1981:47: 2595-602.

Requests for reprints to: Dr JWB Bradfield, Department of Pathology, Medical School, University Walk, Bristol BS8 1TD, England. 